\title{
Decreased energetics in murine hearts bearing the R92Q mutation in cardiac troponin $T$
}

\author{
Maryam M. Javadpour, ${ }^{1}$ Jil C. Tardiff, ${ }^{2}$ Ilka Pinz, ${ }^{1}$ and Joanne S. Ingwall ${ }^{1}$ \\ ${ }^{1}$ NMR Laboratory for Physiological Chemistry, Division of Cardiovascular Medicine, Department of Medicine, \\ Brigham and Women's Hospital, Harvard Medical School, Boston, Massachusetts, USA \\ ${ }^{2}$ Department of Medicine and Department of Physiology and Biophysics, Albert Einstein College of Medicine, Bronx, \\ New York, USA
}

\begin{abstract}
The thin filament protein cardiac troponin $\mathrm{T}(\mathrm{cTnT})$ is an important regulator of myofilament activation. Here we report a significant change in cardiac energetics in transgenic mice bearing the missense mutation $\mathrm{R} 92 \mathrm{Q}$ within the tropomyosin-binding domain of $\mathrm{cTnT}$, a mutation associated with a clinically severe form of familial hypertrophic cardiomyopathy. This functional domain of $\mathrm{cTnT}$ has recently been shown to be a crucial modulator of contractile function despite the fact that it does not directly interact with the ATP hydrolysis site in the myosin head. Simultaneous measurements of cardiac energetics using ${ }^{31} \mathrm{P}$ NMR spectroscopy and contractile performance of the intact beating heart revealed both a decrease in the free energy of ATP hydrolysis available to support contractile work and a marked inability to increase contractile performance upon acute inotropic challenge in hearts from R92Q mice. These results show that alterations in thin filament protein structure and function can lead to significant defects in myocardial energetics and contractile reserve.
\end{abstract}

J. Clin. Invest. 112:768-775 (2003). doi:10.1172/JCI200315967.

\begin{abstract}
Introduction
Hypertrophic cardiomyopathy is a disease characterized by the presence of a nondilated, usually hypertrophied left ventricle (LV) and greater susceptibility to arrhythmias and sudden death. A dominant feature of most patients with this disease is abnormal diastolic function due to impaired relaxation and reduced LV compliance in spite of preserved or even hyperdynamic LV systolic function (1). In about $60 \%$ of these patients, the disease is inherited as an autosomaldominant familial hypertrophic cardiomyopathy (FHC) (2). Most of the FHC mutations affect the proteins in the sarcomere. One of the most clinically malignant FHC mutations is a missense mutation in the gene for the thin-filament protein cardiac troponin $\mathrm{T}(\mathrm{c} \operatorname{Tn} \mathrm{T})$ that results in an amino acid exchange of glutamine (an uncharged amino acid) for
\end{abstract}

Received for publication May 21, 2002, and accepted in revised form May 20, 2003.

Address correspondence to: Jil C. Tardiff, Cardiology Division, Albert Einstein College of Medicine, Section of Molecular Cardiology, Forcheimer G-46, Bronx, New York, 10461 USA. Phone: (718) 430-8914; Fax: (718) 430-8989;

E-mail: tardiff@aecom.yu.edu.

Conflict of interest: The authors have declared that no conflict of interest exists.

Nonstandard abbreviations used: left ventricle (LV); familial hypertrophic cardiomyopathy (FHC); cardiac troponin T (cTnT); tropomyosin $(\mathrm{TM})$; free energy of ATP hydrolysis $\left(\Delta \mathrm{G}_{\sim \mathrm{ATP}}\right)$; inorganic phosphate $(\mathrm{Pi})$; phosphocreatine $(\mathrm{PCr})$; nontransgenic (NTG); LV developed pressure (DevP); rate pressure product (RPP); myocardial oxygen consumption $\left(\mathrm{MVO}_{2}\right)$; sarcoplasmic reticular $\mathrm{Ca}^{2+}$-ATPase (SERCA) arginine (a positively charged amino acid) at residue 92 (R92Q). Individuals with this mutation represent a unique clinical subset of FHC characterized by early sudden death in the absence of overt ventricular hypertrophy; approximately $50 \%$ of affected individuals die by the third decade of life.

Troponin $T$ plays a crucial role in the regulation of the contractile cycle because it binds to tropomyosin (TM) where the two TM polypeptide chains overlap (3-5). Recent biophysical data (6) have shown that when the TnT tail binds to this region, changes in the structure of the C-terminal domain of TM occur that affect the flexibility of the entire TM filament. This in turn determines the affinity of the TM-TnT complex for actin. Two recent reports, one using cTnT (3) and the other skeletal muscle TnT (4), have found that in the absence of the other regulatory thin filament proteins, the tail portion of TnT exhibits a multifunctional role in that it inhibits myosin subfragment- 1 ATPase activity, slows in vitro motility of actin-TM filaments on heavy meromyosin, and increases the cooperativity of myosin subfragment- 1 binding to thin filaments. Thus, in addition to a structural role, TnT plays an important functional role in thin filament activation and crossbridge cycling in striated muscles. Several authors have suggested that the differing structures of the tail domain of TnT's explain the differences between skeletal and cardiac thin filament properties $(4,7)$, differences in the properties of $\operatorname{Tn} \mathrm{T}$ isoforms (8), and differences in properties of the sarcomeres in hypertrophic and dilated cardiomyopathic hearts (9). The R92Q mutation studied here is located 
in the elongated tail domain of $\mathrm{cTnT}$ at the critical site of overlap of the TM monomers, and missense mutations at this site in the cTnT tail have been shown to alter thin filament function $(6,10)$.

The conformational changes that occur in normal thick and thin filament proteins during the contractile cycle are energetically unfavorable and must be coupled to the hydrolysis of ATP in order to proceed. The chemical driving force available to drive ATPase reactions is quantitatively expressed as the free energy of ATP hydrolysis, $\Delta \mathrm{G}_{\sim \mathrm{ATP}}$. The free energy of ATP hydrolysis is the sum of the constant value for ATP hydrolysis under standard conditions corrected for the actual concentrations of ATP, ADP, and inorganic phosphate $(\mathrm{Pi})$ in the cytosol. The relationship between $\Delta \mathrm{G}_{\sim \text { ATP }}$ and contractile performance in the normal myocardium shows that decreasing the chemical driving force for ATPase reactions limits contractile performance (11). Diastolic performance in normal (12) and hypertrophied (13) myocardium is impaired by the accumulation of the hydrolysis products of ATP, namely ADP and Pi, and thus leads to a lower chemical driving force.

While it is now clear that the concentration of ATP (as well as the primary high-energy reserve compound phosphocreatine [PCr]) is lower in the severely failing human heart than for normal heart (14), little information is available defining the energetics of hearts of either FHC patients or animal models of FHC. One study using ${ }^{31} \mathrm{P}$ NMR spectroscopy of hearts of asymptomatic patients with hypertrophic cardiomyopathy showed the ratio of PCr to ATP concentrations was decreased (15). A ${ }^{31}$ P NMR spectroscopy study of hearts isolated from a mouse bearing a missense mutation at the 403 position of myosin heavy chain made the unexpected observation that this mutation in the actinbinding domain of myosin led to decreased PCr concentrations and less favorable $\Delta \mathrm{G}_{\sim \mathrm{ATP}}(16)$. These hearts demonstrated diastolic dysfunction and reduced contractile reserve with inotropic challenge.

In the present study using the intact heart, we test the hypothesis that the structural changes caused by the R92Q missense mutation in the TM-binding domain of cTnT lead to increased ATP utilization and impair the ability of the heart to recruit its contractile reserve upon acute inotropic challenge. If this is the case, it would be the first time that a mutation in a thin filament protein is shown to result in altered energetics in the intact heart. We used a well-characterized mouse model that expresses a $c$-myc-tagged full-length murine cTnT bearing the R92Q mutation (67\% replacement of native cTnT) specifically in the heart (17). This model was chosen because it recapitulates many of the aspects of the human disease including myocyte disarray and diastolic dysfunction in the absence of ventricular hypertrophy. We used the noninvasive tool of ${ }^{31} \mathrm{P}$ NMR spectroscopy to measure the energetic state of the heart simultaneously with measurements of systolic and diastolic contractile performance (18). We found that the presence of the sin- gle amino acid missense mutation R92Q in cTnT, known to occur within the CTnT-TM binding site that determines thin filament structure and function, leads to increased ATP utilization and impaired ability of the heart to recruit its contractile reserve.

\section{Methods}

Mice. Sixteen-week-old mice bearing c-myc-tagged murine cTnT with the R92Q mutation (R92Q) or WT cTnT with the c-myc tag (WT-Tg) were generated as previously described (17); sibling mice were used as nontransgenic (NTG) controls. Each animal was genotyped by PCR-amplified tail DNA and restriction enzyme digestion to confirm the presence or absence of the mutation. Animals were maintained in accordance with NIH guidelines for the care and use of laboratory animals. The experimental protocol was approved by the Standing Committee on Animals of Harvard Medical Area and followed current NIH and American Physiologic Society guidelines.

Isolated perfused heart preparation. Hearts with cardiacspecific R92Q mutation in cTnT (R92Q) $(n=15)$ and their NTG littermate controls $(n=12)$, and hearts with normal cTnT bearing the $c-m y c$ tag (WT-Tg) $(n=4)$ and their littermate controls $(n=4)$ were isolated and perfused in the Langendorff mode as described (19). Hearts were initially perfused with a phosphate-free Krebs-Henseleit buffer containing $118 \mathrm{mM} \mathrm{NaCl}, 5.3$ $\mathrm{mM} \mathrm{KCl}, 2.5 \mathrm{mM} \mathrm{CaCl}_{2}, 1.2 \mathrm{mM} \mathrm{MgSO} 4,0.5 \mathrm{mM}$ EDTA, $25 \mathrm{mM} \mathrm{NaHCO}$, and $11 \mathrm{mM}$ glucose equilibrated with $95 \% \mathrm{O}_{2}$ and $5 \% \mathrm{CO}_{2}, \mathrm{pH}$ 7.4. A water-filled balloon custom-made of polyvinylchloride film was inserted through the mitral valve into the $L V$ via an incision in the left atrium. The balloon was connected to a pressure transducer (Statham P23Db; Gould Statham Inc., Medical Provision Department, Oxnard, California, USA) for continuous recording of LV pressures and heart rate. The size of the balloon matched the size of the ventricular cavity. The balloon was inflated to set LV end diastolic pressure to approximately $10 \mathrm{mmHg}$ and the balloon volume was then held constant. Isovolumic contractile performance data were collected online at a sampling rate of $200 \mathrm{~Hz}$ using a commercially available data acquisition system (MacLab; ADInstruments Pty., Milford, Massachusetts, USA). LV developed pressure (DevP), i.e., the difference between systolic pressure and end diastolic pressure, and the minimum and maximum values within a beat of the first derivative of left ventricular pressure $(+\mathrm{dP} / \mathrm{dt}$ and $-\mathrm{dP} / \mathrm{dt})$ were calculated offline. All hearts were paced at $7 \mathrm{~Hz}$ using monophasic square-wave pulses delivered from a stimulator (model S88; Grass Instrument Co., Quincy, Massachusetts, USA) through salt-bridge pacing wires consisting of PE-90 tubing filled with $4 \mathrm{M} \mathrm{KCl}$ in $2 \%$ agarose.

Experimental protocols. In one protocol, ${ }^{31} \mathrm{P}$ NMR spectra of the whole heart (eight sibling NTG and nine R92Q hearts and four each sibling NTG and WT-Tg hearts) were obtained during baseline perfusion with 
buffer containing only glucose. The buffer was then switched to one containing both glucose and pyruvate $(0.5 \mathrm{mM})$ and another ${ }^{31} \mathrm{P}$ NMR spectrum was obtained. Adding pyruvate to the buffer maximized the ratio of PCr to ATP. Finally hearts were studied at high workload achieved by increasing the total $\left[\mathrm{Ca}^{2+}\right]$ to 4.0 $\mathrm{mM}$ in a perfusate buffer containing both glucose and pyruvate. Data defining isovolumic contractile performance were collected continuously throughout the three sequential 16-minute periods required for these NMR measurements. In the second protocol, myocardial oxygen consumption was measured in four NTG and five $\mathrm{R} 92 \mathrm{Q}$ isolated perfused hearts as described (20). At the end of the experiments, each heart was either rapidly weighed or freeze-clamped, and then stored at $-80^{\circ} \mathrm{C}$ for subsequent biochemical assays.

${ }^{31} \mathrm{P}$ NMR spectroscopy. ${ }^{31} \mathrm{P}$ NMR spectra were obtained at $161.94 \mathrm{MHz}$ using a GE-400 wide-bore spectrometer (Omega, General Electric, Fremont, California, USA). Hearts were placed in a $10-\mathrm{mm}$ glass NMR tube and inserted into a custom-made ${ }^{1} \mathrm{H} /{ }^{31} \mathrm{P}$ double-tuned probe situated in a superconducting magnet $(89-\mathrm{mm}$ bore, $9.4 \mathrm{~T}$ ). Spectra were collected without proton decoupling at a pulse width of $16 \mu \mathrm{s}$, pulse angle of $60^{\circ} \mathrm{C}$, recycling time of 2.14 seconds, and sweep width of $6,000 \mathrm{~Hz}$. Spectra for individual hearts were obtained by signal averaging 416 free induction decays; the spectra shown in Figure 1 are the sum of six such spectra. Spectra were analyzed using $20-\mathrm{Hz}$ exponential multiplication and zero and first-order phase corrections. The resonance areas corresponding to ATP, PCr, and Pi were fitted to Lorentzian functions calculated using the commercially available program NMR1 (NMR1, Syracuse, New York, USA) (21) and corrected for saturation [ATP (1.0), PCr (1.2), and Pi (1.15)].

Biochemical measurements. Five to $10 \mathrm{mg}$ of $\mathrm{LV}$ tissue from NTG and R92Q hearts was homogenized for 10 seconds at $4^{\circ} \mathrm{C}$ in potassium phosphate buffer containing $1 \mathrm{mM}$ EDTA and $1 \mathrm{mM} \beta$-mercaptoethanol, $\mathrm{pH} 7.4$ (final concentration, $5 \mathrm{mg}$ tissue $/ \mathrm{ml}$ ). Aliquots were removed for assays of protein (22) using BSA as the standard and for total creatine content (23). Triton $\mathrm{X}-100$ was then added to the homogenate at a final concentration of $0.1 \%$. Tissue activities of creatine kinase; the glycolytic enzymes phosphofructokinase, GAPDH, and lactate dehydrogenase; the mitochondrial enzyme citrate synthase and the isoenzyme distributions of creatine kinase and lactate dehydrogenase were measured as described (24-27). All reagents came from Sigma Chemical Co. (St. Louis, Missouri, USA) and were at least analytical grade.

Metabolite concentrations. To determine the cytosolic concentration of ATP, the absolute resonance areas corresponding to ATP (average of $\beta$-phosphate and $\gamma$-phosphate resonance areas) in the ${ }^{31} \mathrm{P}$ NMR spectra during baseline perfusion were normalized by heart weight. Mean ATP area units/mg wet weight for NTG and R92Q hearts were $0.63 \pm 0.04$ and $0.58 \pm 0.08$ during glucose perfusion and $0.55 \pm 0.03$ and $0.50 \pm 0.07$ for perfusion with glucose and pyruvate, respectively. We made the assumption that the ratio of intracellular volume to cardiac mass of $0.48 \mu \mathrm{l} / \mathrm{mg}$ wet weight was the same for all hearts (28). Thus, regardless of the buffer used, the NTG and R92Q hearts have indistinguishable [ATP].

Cytosolic $[\mathrm{ADP}]$ was calculated using the equilibrium expression for the creatine kinase reaction and values for ATP, PCr, creatine, and $\mathrm{H}^{+}$concentrations obtained by NMR spectroscopy and biochemical assays: $[\mathrm{ADP}]=([\mathrm{ATP}][$ free creatine $]) /\left([\mathrm{PCr}]\left[\mathrm{H}^{+}\right] K_{\mathrm{eq}}\right)$; where $K_{\mathrm{eq}}$ is $1.66 \times 10^{9} \mathrm{M}^{-1}$ for $\left[\mathrm{Mg}^{2+}\right]$ of $1.0 \mathrm{mM}(29,30)$.

The free energy released by ATP hydrolysis $\left(\Delta \mathrm{G}_{\sim \mathrm{ATP}}\right)$ drives the ATPase reactions in the cell. Because $\Delta \mathrm{G}_{\sim \mathrm{ATP}}$ is a negative number, we describe changes in $\Delta \mathrm{G}_{\sim \mathrm{ATP}}$ in absolute values, denoted as $\left|\Delta \mathrm{G}_{\sim \mathrm{ATP}}\right| . \Delta \mathrm{G}_{\sim \mathrm{ATP}}$ is calculated as $\left|\Delta \mathrm{G}_{\sim \mathrm{ATP}}\right|(\mathrm{kJ} / \mathrm{mol})=\left|\Delta \mathrm{G}^{\circ}+R T \ln ([\mathrm{ADP}][\mathrm{Pi}] /[\mathrm{ATP}])\right|$ where $\Delta \mathrm{G}^{\circ}(-30.5 \mathrm{~kJ} / \mathrm{mol})$ is the value of $\Delta \mathrm{G}_{\sim \mathrm{ATP}}$ under standard conditions of molarity, temperature, $\mathrm{pH}$, and $\left[\mathrm{Mg}^{2+}\right](28), R$ is the gas constant $(8.3 \mathrm{~J} / \mathrm{mol} \mathrm{K})$, and $T$ is the temperature in degrees Kelvin.

Statistical analysis. All results are expressed as mean \pm SEM. The paired (for repeated measures performed on the same heart) or unpaired Student $t$ test or factorial ANOVA was used to compare the NTG and transgenic hearts as appropriate. All statistical analyses were performed with STATVIEW (Brain Power Inc., Calabasas, California, USA), and a value of $P<0.05$ was considered significant.

\section{Results}

Characteristics of mice. NTG and R92Q transgenic littermates had similar body weights $(26.2 \pm 0.6$ vs. $27.2 \pm 0.7$ $\mathrm{g}$, respectively). The atria of $\mathrm{R} 92 \mathrm{Q}$ hearts were larger (NTG, $7.2 \pm 0.6$ vs. R92Q, $10.2 \pm 0.6 \mathrm{mg}$ ), while both the right ventricular (NTG, $20.1 \pm 1.3$ vs. R92Q, $17.3 \pm 10.8$ $\mathrm{mg}$ ) and $\mathrm{LV}$ (NTG, $91.7 \pm 4.2$ vs. R92Q, $73.6 \pm 35 \mathrm{mg}$ ) weights of R92Q hearts were smaller than those of NTG hearts. Because the overall heart weight of the R92Q hearts was about $15 \%$ less than that of NTG hearts (NTG, $119.1 \pm 5.4$ vs. R92Q, $100.9 \pm 3.9 \mathrm{mg}$ ), the

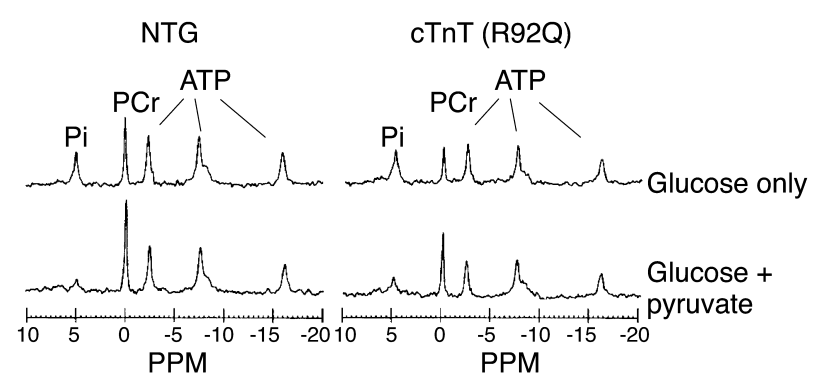

\section{Figure 1}

Sum of ${ }^{31}$ P NMR spectra from six 16-week-old NTG (left) and R92Q (right) hearts obtained during baseline perfusion with buffer containing either $11 \mathrm{mM}$ glucose (top spectra) or $11 \mathrm{mM}$ glucose with $0.5 \mathrm{mM}$ pyruvate (bottom spectra). Resonance areas from left to right correspond to $\mathrm{Pi}, \mathrm{PCr}$, and $\gamma-, \alpha$-, and $\beta$-phosphates of ATP. $\mathrm{R} 29 \mathrm{Q}$ hearts had lower $\mathrm{PCr}$ and higher Pi resonance areas than did NTG hearts. PPM, parts per million. 
Table 1

Indices of isovolumic contractile performance of NTG and cTnT (R92Q) hearts at baseline and in response to inotropic stimulation

\begin{tabular}{|c|c|c|c|c|}
\hline & \multicolumn{2}{|c|}{ NTG } & \multicolumn{2}{|c|}{ R92Q } \\
\hline & {$\left[\mathrm{Ca}^{2+}\right]=2.5 \mathrm{mM}$} & {$\left[\mathrm{Ca}^{2+}\right]=4.0 \mathrm{mM}$} & {$\left[\mathrm{Ca}^{2+}\right]=2.5 \mathrm{mM}$} & {$\left[\mathrm{Ca}^{2+}\right]=4.0 \mathrm{mM}$} \\
\hline$n$ & 8 & 7 & 9 & 9 \\
\hline SP & $94.4 \pm 5.9$ & $141.5 \pm 10^{\mathrm{A}}$ & $83.9 \pm 9.2^{\mathrm{B}}$ & $86.3 \pm 12.6^{B}$ \\
\hline EDP & $9.9 \pm 0.6$ & $9.4 \pm 1.1$ & $9.7 \pm 0.3$ & $11.1 \pm 0.8$ \\
\hline$+\mathrm{dP} / \mathrm{dt}$ & $3,500 \pm 220$ & $5,500 \pm 500^{A}$ & $3,400 \pm 470^{\mathrm{B}}$ & $3,900 \pm 700^{B}$ \\
\hline$-d P / d t$ & $2,700 \pm 250$ & $4,900 \pm 360^{A}$ & $2,000 \pm 300^{A, B}$ & $2,300 \pm 440^{B}$ \\
\hline DevP & $84.4 \pm 6.0$ & $132.1 \pm 9.7^{A}$ & $74.3 \pm 9.3^{B}$ & $75.2 \pm 12.5^{B}$ \\
\hline $\mathrm{RPP} \times 10^{-3}$ & $39.1 \pm 2.7$ & $59.2 \pm 2.8^{A}$ & $34.7 \pm 4.3^{B}$ & $33.6 \pm 5.3^{B}$ \\
\hline
\end{tabular}

NTG and cTnT (R92Q) hearts were perfused with buffer containing glucose and pyruvate, and contractile performance was measured at baseline (2.5 mM calcium) and in response to inotropic stimulation with $4.0 \mathrm{mM}$ calcium. ${ }^{A} P<0.05$ compared with NTG baseline. ${ }^{\mathrm{B} P}<0.05$ vs. NTG high work. Heart rate equals 460 beats per minute. EDP, end diastolic pressure. SP (systolic pressure), EDP, and DevP are given in $\mathrm{mmHg} ; \mathrm{dP} / \mathrm{dt}$ is in $\mathrm{mmHg} / \mathrm{s} ; \mathrm{RPP}$ is in $\mathrm{mmHg} / \mathrm{min}$.

heart weight to body weight ratio of the R92Q mice was about $20 \%$ smaller (NTG, $4.6 \pm 0.2$ vs. R92Q, $3.7 \pm 0.1$ ). Thus, in agreement with previous reports (17), the transgenic hearts bearing the R92Q mutation in cTnT were smaller than sibling NTG hearts. Hearts bearing the c-myc tag on WT cTnT (WT-Tg) were not different in weight from sibling NTG hearts $(129 \pm 8.1$ vs. $113 \pm 5.9 \mathrm{mg}$ for WT-Tg and NTG mice, both weighing 25.6 g, respectively).

Contractile performance. When perfused in the Langendorff mode (Table 1), murine hearts bearing the R92Q mutation in $\mathrm{cTnT}$ exhibited similar indices of systolic contractile performance (assessed as systolic pressure, peak DevP, $+\mathrm{dP} / \mathrm{dt}$, and rate pressure product [RPP]) as NTG hearts. Diastolic function (assessed as $-\mathrm{dP} / \mathrm{dt}$ ) was impaired in $\mathrm{R} 92 \mathrm{Q}$ hearts.

To determine whether R92Q hearts were able to increase contractile performance in response to inotropic challenge, we measured contractile performance in sibling NTG and R92Q hearts perfused with normal and high $\left[\mathrm{Ca}^{2+}\right]$ (Table 1). In the NTG hearts, increasing $\left[\mathrm{Ca}^{2+}\right]$ from 2.5 to $4 \mathrm{mM}$ resulted in a greater than $50 \%$ increase in all indices of contractile performance: systolic pressure, peak DevP, and $+\mathrm{dP} / \mathrm{dt}$. The rate of relaxation also increased. In contrast, the $\mathrm{R} 92 \mathrm{Q}$ hearts failed to increase contractile performance in response to increases in $\left[\mathrm{Ca}^{2+}\right]$; none of the indices of either systolic or diastolic performance changed. Thus, transgenic hearts bearing the R92Q mutation in cTnT exhibit diastolic dysfunction under baseline conditions and fail to increase contractile performance in response to inotropic challenge.

Energetics. We used ${ }^{31} \mathrm{P}$ NMR spectroscopy to measure ATP, PCr, and Pi concentrations of sibling NTG and $\mathrm{R} 92 \mathrm{Q}$ hearts at baseline and at high $\left[\mathrm{Ca}^{2+}\right]$ to determine whether the R92Q mutation in $\mathrm{CTnT}$ causes a change in energetics at baseline and to test whether the decreases in contractile reserve are due to decreases in energy reserve (12). We also made comparable measurements in sibling NTG and WT-Tg hearts to test whether the presence of the $c-m y c$ tag alone affected whole-heart energetics.

Figure 1 shows ${ }^{31} \mathrm{P}$ NMR spectra acquired during baseline perfusion from NTG and R92Q hearts per- fused with either glucose only (top) or glucose plus pyruvate (bottom). The ATP resonance areas/mg wet weight, and thus the amount of ATP/mg tissue, were similar for the NTG and R92Q hearts, but the PCr resonance areas were smaller and Pi resonance areas higher in the R92Q heart, regardless of the exogenous oxidizable substrate supplied to the heart.

Table 2 and Table 3 show the mean values of [ATP], [ADP], [PCr], and [Pi] determined at baseline with glucose-containing perfusate and at baseline and high workload conditions when both glucose and pyruvate were the exogenous substrates supplied to support ATP synthesis for NTG and R92Q hearts. Regardless of exogenous substrate supplied to support ATP synthesis, NTG and R92Q hearts had similar [ATP] but R92Q hearts had lower [PCr] (by 20\%) and higher [Pi] (2.2- to 3.7-fold). The decrease in [PCr] in $\mathrm{R} 92 \mathrm{Q}$ hearts was not due to a decrease in total creatine concentration, which was not different between NTG and R92Q hearts (Table 4). Instead, the decrease in [PCr] is due to increased ATP utilization. R92Q hearts had higher cytosolic [ADP] and higher [Pi] than NTG hearts. Intracellular $\mathrm{pH}$ was similar in NTG and R92Q hearts and averaged 7.14.

Changes in [ATP], [ADP], and [Pi] can be expressed as a single number, the free energy of ATP hydrolysis, $\Delta \mathrm{G}_{\sim \text { ATP. }}$. This value represents the driving force for the ATPase reactions in the cell and describes the energy state of the cell. Regardless of which substrate was sup-

\section{Table 2}

Energetics of NTG and cTnT (R92Q) hearts perfused with buffer containing glucose and $2.5 \mathrm{mM} \mathrm{Ca}^{2+}$

$\begin{array}{lcc} & \text { NTG } & \text { R92Q } \\ n & 8 & 10 \\ \operatorname{ATP}(\mathrm{mM}) & 9.9 \pm 0.01 & 10.0 \pm 0.02 \\ \mathrm{PCr}(\mathrm{mM}) & 10.7 \pm 0.7 & 8.7 \pm 1.0 \\ \mathrm{Pi}(\mathrm{mM}) & 7.9 \pm 1.1 & 17.2 \pm 2.4^{\mathrm{A}} \\ \mathrm{PH} & 7.14 \pm 0.01 & 7.15 \pm 0.02 \\ \mathrm{ADP}(\mu \mathrm{M}) & 136 \pm 18 & 233 \pm 50^{\mathrm{A}} \\ \left|\Delta \mathrm{G}_{\sim \text { ATP }}\right|(\mathrm{kJ} / \mathrm{mol}) & 54.3 \pm 0.6 & 51.3 \pm 0.5^{\mathrm{A}}\end{array}$

${ }^{A} P<0.05$ compared with NTG. 
Energetics of NTG and CTnT (R92Q) hearts perfused with glucose and pyruvate at baseline and in response to inotropic stimulus

\begin{tabular}{|c|c|c|c|c|}
\hline & \multicolumn{2}{|c|}{ NTG } & \multicolumn{2}{|c|}{ R92Q } \\
\hline & {$\left[\mathrm{Ca}^{2+}\right]=2.5 \mathrm{mM}$} & {$\left[\mathrm{Ca}^{2+}\right]=4.0 \mathrm{mM}$} & {$\left[\mathrm{Ca}^{2+}\right]=2.5 \mathrm{mM}$} & {$\left[\mathrm{Ca}^{2+}\right]=4.0 \mathrm{mM}$} \\
\hline$n$ & 7 & 6 & 9 & 8 \\
\hline ATP $(\mathrm{mM})$ & $8.8 \pm 0.3$ & $8.8 \pm 0.3$ & $9.4 \pm 0.6$ & $8.1 \pm 0.6^{C}$ \\
\hline $\mathrm{PCr}(\mathrm{mM})$ & $14.7 \pm 0.6$ & $11.7 \pm 0.6^{A}$ & $12.3 \pm 0.3^{\mathrm{A}}$ & $10.0 \pm 0.9^{\mathrm{A}, \mathrm{C}}$ \\
\hline $\mathrm{Pi}(\mathrm{mM})$ & $2.7 \pm 0.5$ & $4.9 \pm 0.5$ & $10.1 \pm 1.8^{\mathrm{A}, \mathrm{B}}$ & $10.8 \pm 0.9^{\mathrm{A}, \mathrm{B}}$ \\
\hline $\mathrm{pH}$ & $7.14 \pm 0.01$ & $7.14 \pm 0.02$ & $7.11 \pm 0.06$ & $7.11 \pm 0.02$ \\
\hline $\operatorname{ADP}(\mu \mathrm{M})$ & $64 \pm 8$ & $113 \pm 25$ & $101 \pm 19$ & $129 \pm 31$ \\
\hline$\left|\Delta \mathrm{G}_{\sim \mathrm{ATP}}\right|(\mathrm{kJ} / \mathrm{mol})$ & $58.9 \pm 0.8$ & $55.9 \pm 0.4^{\mathrm{A}}$ & $54.8 \pm 0.7^{A}$ & $53.4 \pm 0.7^{\mathrm{A}, \mathrm{B}, \mathrm{C}}$ \\
\hline
\end{tabular}

plied to the heart to support ATP synthesis, the values of $\left|\Delta \mathrm{G}_{\sim \mathrm{ATP}}\right|$ are approximately 3-4 $\mathrm{kJ} / \mathrm{mol}$ lower in R92Q hearts. Thus, hearts bearing the R92Q cTnT missense mutation have a lower driving force for ATPase reactions. The presence of the $c-m y c$ tag did not change the driving force for the ATPase reactions regardless of exogenous substrate supplied to the hearts: values for $\left|\Delta G_{\sim A T P}\right|$ for WT-Tg and sibling NTG hearts perfused with glucose and pyruvate were 60.7 and $60.8 \mathrm{~kJ} / \mathrm{mol}$, respectively.

Contractile performance and energetics. The relationships between physiologic performance of the heart assessed as RPP and $\left|\Delta \mathrm{G}_{\sim \mathrm{ATP}}\right|$ for NTG, WT-Tg, and R92Q hearts perfused using buffers containing 2.5 and $4.0 \mathrm{mM}$ $\left[\mathrm{Ca}^{2+}\right]$ are shown in Figure 2. Both NTG and WT-Tg hearts demonstrated the expected decrease in $\left|\Delta \mathrm{G}_{\sim \mathrm{ATP}}\right|$ as RPP increased in response to inotropic challenge (12, 16), and the quantitative relationships between RPP and $\left|\Delta \mathrm{G}_{\sim \mathrm{ATP}}\right|$ were indistinguishable for NTG and for WT$\mathrm{Tg}$ hearts. In contrast, R92Q hearts not only had lower $\left|\Delta \mathrm{G}_{\sim \mathrm{ATP}}\right|$ at baseline conditions but failed to support an increase in RPP with the equivalent inotropic challenge. Upon inotropic challenge, the free energy of ATP hydrolysis for R92Q hearts fell by about $1.4 \mathrm{~kJ} / \mathrm{mol}$ and RPP did not increase. Thus the R92Q hearts were not able to transduce the small amount of free energy released from ATP hydrolysis into mechanical work.

To rule out that the inability of R92Q hearts to increase contractile performance was due to decreased capacity for ATP synthesis, we also measured myocardial oxygen consumption $\left(\mathrm{MVO}_{2}\right)$ at different RPPs, which were varied by changing perfusate $\left[\mathrm{Ca}^{2+}\right]$ (Figure 3 ). The data for four NTG and five R92Q hearts were fit well by the same linear relationship: $\mathrm{MVO}_{2}=1.6 \times 10^{-5}$ $\mathrm{RPP}+4.7$. The capacity for ATP synthesis via oxidative phosphorylation assessed by citrate synthase activity (a good marker of mitochondrial mass), via glycolysis (measured as activities of phosphofructokinase, GAPDH, and lactate dehydrogenase), and via the creatine kinase reaction was the same in R92Q and NTG hearts (Table 4). The isoenzyme distributions for lactate dehydrogenase and creatine kinase were unchanged (data not shown). In addition, the capacity for ATP synthesis was unchanged in WT-Tg hearts (data not shown). Thus, neither the capacity for ATP synthesis nor the coupling of $\mathrm{MVO}_{2}$ and RPP differed between NTG and R92Q hearts.

\section{Discussion}

There are two major findings in the studies reported here. First, hearts bearing the R92Q mutation in cTnT have decreased [PCr] and increased [Pi] and [ADP], leading to a large decrease $(\sim 4 \mathrm{~kJ} / \mathrm{mol})$ in the free energy of ATP hydrolysis, $\left|\Delta \mathrm{G}_{\sim \mathrm{ATP}}\right|$. This result shows that this single amino acid replacement in the TM-binding domain of $\mathrm{cTnT}$ at a site known to cause changes in thin filament structure and function is sufficient to increase ATP utilization in the intact beating heart. Second, upon acute inotropic challenge, the small amount of free energy expended in the cTnT mutant hearts $(\sim 1.4 \mathrm{~kJ} / \mathrm{mol})$ failed to support an increase in contractile performance. The observation that R92Q hearts fail to recruit their contractile reserve suggests that the R92Q mutation in the TM-binding domain of cTnT disrupts the normal transmission of the $\mathrm{Ca}^{2+}$ signaling cascade from troponin $\mathrm{C}(\mathrm{c} \operatorname{TnC})$ to TM. Mutant $\mathrm{cTn} T$ hearts also exhibit work-induced diastolic dysfunction. Importantly, since hearts (and myocytes) bearing the cardiac-specific R92Q mutation are smaller than littermate NTG hearts, these energetic and contractile

\section{Table 4}

Biochemical characteristics of NTG and cTnT (R92Q) mouse hearts

$\begin{array}{lcc} & \text { NTG } & \text { R92Q } \\ n & 8 & 8 \\ \text { Protein content } & 0.173 \pm 0.008 & 0.174 \pm 0.004 \\ \text { (mg/mg wet weight) } & & \\ {[\mathrm{Cr}](\mathrm{mM})} & 21.56 \pm 1.0 & 21.2 \pm 1.99 \\ \mathrm{CK}(\mathrm{IU} / \mathrm{mg} \text { protein) } & 8.0 \pm 0.2 & 8.7 \pm 0.4 \\ \mathrm{LDH}(\mathrm{IU} / \mathrm{mg} \text { protein }) & 0.93 \pm 0.03 & 1.0 \pm 0.06 \\ \mathrm{GAPDH}(\mathrm{mlU} / \mathrm{mg} \text { protein }) & 940 \pm 78 & 919 \pm 59 \\ \mathrm{PFK}(\mathrm{mlU} / \mathrm{mg} \text { protein }) & 315 \pm 23 & 316 \pm 23 \\ \mathrm{CS}(\mathrm{mIU} / \mathrm{mg} \text { protein) } & 805 \pm 37 & 799 \pm 45\end{array}$

Results for NTG and R92Q hearts are indistinguishable. Results for c-myctagged WT and sibling NTG hearts are also indistinguishable (data not shown). $\mathrm{Cr}$, creatine; PFK, phosphofructokinase; LDH, lactate dehydrogenase; $\mathrm{CS}$, citrate synthase. Data are mean $\pm \mathrm{SEM}$. 


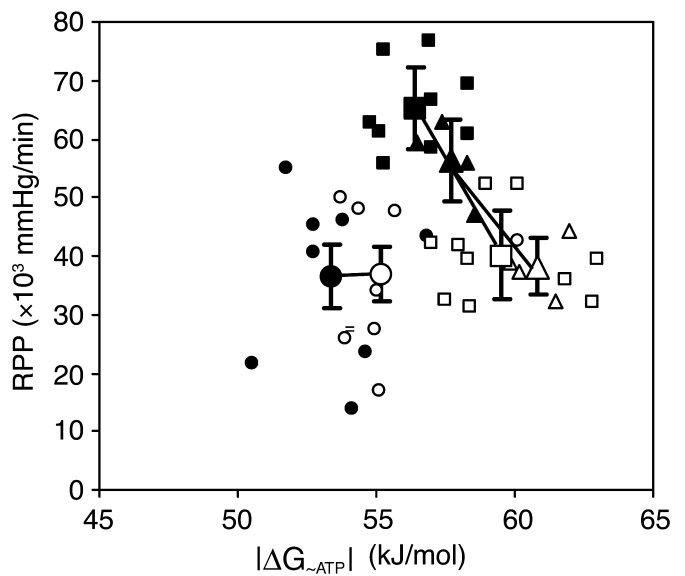

Figure 2

Plot of the relationship between RPP and $\left|\Delta \mathrm{G}_{\sim \text { ATP }}\right|$ for NTG (squares, $n=9$ ), WT-Tg (triangles, $n=4$ ), and R92Q (circles, $n=8$ ) hearts perfused with buffers containing glucose and pyruvate with $2.5 \mathrm{mM}$ (open symbols) and $4.0 \mathrm{mM}$ (filled symbols) $\mathrm{Ca}^{2+}$. The means of the NTG hearts (large squares), WT-Tg hearts (large triangles), and R92Q hearts (large circles) for each workload are connected. The relationships for NTG and WT-Tg mice are indistinguishable; the relationship for R92Q hearts differs from that of both NTG and WT-Tg hearts.

defects are not a consequence of cardiac hypertrophy or $\mathrm{LV}$ dilatation but are the result of the mutation in the thin filament protein cTnT.

The known molecular mechanisms that can lead to decreased $\mathrm{PCr}$ with normal total creatine and increased Pi and ADP concentrations leading to lower $\left|\Delta \mathrm{G}_{\sim \mathrm{ATP}}\right|$ include: increased use of myofibrillar ATP (by far the major ATPase in the myocyte); a very large increase in ATP utilization by the sarcoplasmic reticular $\mathrm{Ca}^{2+}$-ATPase (SERCA); and decreased ATP synthesis, singly or in any combination (31). The possibility that the R92Q mutation in $\mathrm{cTnT}$ leads to a change in the conformation of $c \mathrm{TnC}$ that in turn leads to increased ATP utilization by SERCA can be ruled out. As noted by Tobacman and colleagues (3), cTnC is physically distant from the cTnT tail. Instead, the physical interaction that occurs is between the cTnT tail and the flexible region of the TM filament, thereby altering the TM-actin interaction, which in turn alters crossbridge cycling. The physical constraints of this highly ordered system must lead to increased ATP utilization by the myofibril, not by SERCA. Additional evidence ruling out increased ATP utilization by SERCA includes the observation that myocytes bearing the R92Q mutation in $\mathrm{CTnT}$ have indistinguishable levels of SERCA mRNA (17) and protein (J.C. Tardiff, unpublished results). Finally, using the same protocol described here, hearts in which SERCA levels varied by as much as $50 \%$ have a normal relationship between RPP and $\left|\Delta G_{\sim \text { ATP }}\right|$ (I. Pinz, R. Tian, W. Dillman, and J.S. Ingwall, unpublished results).

Decreased free energy of ATP hydrolysis and the inability of the R92Q heart to increase contractile performance upon demand are not due to decreased ATP synthesis since none of the following differed in R92Q and NTG hearts: ATP and total creatine concentrations; the capacity for ATP synthesis by oxidative phosphorylation, glycolysis, or via the creatine kinase reaction; and the $\mathrm{MVO}_{2} / \mathrm{RPP}$ relationship. In addition, the changes in $\left|\Delta G_{\sim A T P}\right|$ were independent of the exogenous substrate supplied.

Thus, the results presented here show that the R92Q mutation in the TM-binding domain of $c \operatorname{TnT}$ changes the cTnT-TM interaction in such a way that whole-heart ATP utilization rates are chronically higher than those of NTG hearts, as evidenced by a decrease in PCr and increases in Pi and ADP concentrations. The magnitude of the difference between cTnT and NTG hearts in the driving force for the ATPase reactions is large $(\sim 4 \mathrm{~kJ} / \mathrm{mol})$ and is particularly surprising in view of the relatively modest effects of the R92Q mutation on the flexibility of the TM filament and affinity for actin compared with the R92L and R92W mutations observed in careful biophysical measurements using isolated polypeptides (6). This illustrates the importance of making these measurements in the intact heart, where the impact of apparently small perturbations at the polypeptide level can be summed over a large number of myofibrils. The magnitude of the difference in the driving forces for the ATPase reaction between R92Q and NTG hearts is comparable to the change in energy reserve that led to decreased contractile reserve in normal hearts (11), and is sufficient to explain the decrease in contractile reserve observed for the R92Q hearts. As reported previously for normal and hypertrophied hearts $(12,13)$, the relatively high concentrations of $\mathrm{Pi}$ and ADP observed for the R92Q hearts are also sufficient to cause the slowed rate of relaxation.

The only other mouse model of FHC in which energetics and contractile performance have been report-

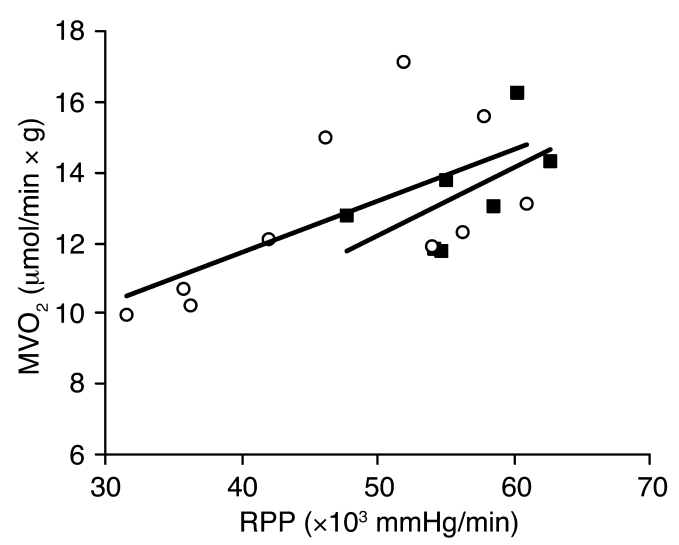

Figure 3

Relationship between $\mathrm{MVO}_{2}$ and RPP in NTG (filled squares) and R92Q (open circles) hearts. Hearts were perfused with the same buffers described in Figure 2 legend. The equations for the linear regressions are similar in both groups: NTG ( $n=4$, seven data points), $\mathrm{MVO}_{2}=1.9 x+2.65 ; \mathrm{R} 92 \mathrm{Q}(n=5$, ten data points $), \mathrm{MVO}_{2}=1.4 x+5.94$ however, the R92Q hearts fall on the lower part of the relationship. 
ed in an intact heart is the R403Q missense mutation (50\% replacement) in the murine $\alpha$-myosin heavy chain gene ( $\alpha \mathrm{MyHC})(16)$. The comparison with the mouse hearts bearing the R92Q mutation in the $c T n T$ gene is instructive. The most striking similarity between the hearts bearing the R403Q mutation in the $\alpha M y H C$ gene and the R92Q mutation in the $c T n T$ gene is the unexpected defects in energetics; both hearts have decreased $\mathrm{PCr}$ and increased $\mathrm{Pi}$ concentrations, leading to a decrease in the free energy of ATP hydrolysis. It is important to emphasize that, although neither amino acid residue is physically near the ATP-hydrolysis site in the myosin head, a common result of both the R403Q mutation in the actinbinding domain of the myosin head and the R92Q mutation in the TM-binding domain of cTnT is greater ATP utilization. Taken together, these results show that altering the actin-myosin interaction either by a charge change in the actin-binding domain of myosin (R403Q) or by altering TM flexibility and affinity for actin via a charge change in cTnT (R92Q) leads to structural changes in the thin filament that cost energy at the level of the cardiac sarcomere. As elegantly shown by others for both cardiac and skeletal muscle TnT's, the tail portion of TnT influences the size of the cooperative unit and the transition from weak to strong binding states, two ways that mutated filaments can alter energy utilization. Of note, in 1998, Sweeney and colleagues suggested that cTnT-related mutations in the $\mathrm{N}$-terminal domain could increase the energy cost of force production; the data presented here have shown for the first time that this is indeed the case (32).

An important difference between the two FHC models is that the $\alpha \mathrm{MyHC}$ mutant mouse heart was able to use the free energy available from ATP hydrolysis to support an increase in contractile performance elicited by the same inotropic challenge used in this study for the cTnT mutant mouse. The change in $\left|\Delta \mathrm{G}_{\sim \mathrm{ATP}}\right|$ for the R403Q $\alpha$ MyHC mouse heart was over $4 \mathrm{~kJ} / \mathrm{mol}$, the same change observed for their (and the present) littermate controls. For the cTnT mouse hearts bearing the R92Q mutation, there was only a small change in $\left|\Delta \mathrm{G}_{\sim \mathrm{ATP}}\right|$ upon challenge with high $\mathrm{Ca}^{2+}$ perfusion $(\sim 1.4 \mathrm{~kJ} / \mathrm{mol})$, which was unable to support an increase in contractile performance. Although speculative, it seems likely that the differences in energetic and contractile phenotypes observed for these two thick and thin filament FHC-associated mutations in these murine models may have a clinical correlate and contribute to the strikingly different clinical courses seen in patients with mutations in different sarcomeric proteins. A recent report using a murine mutant cTnT model $(\mathrm{I} 79 \mathrm{~N})$ with characteristics similar to the R92Q mutation studied here demonstrated that while the I79N mice were able to tolerate a training exercise regimen, mice subjected to intraperitoneal isoproterenol injection exhibited a high frequency of sudden death (33). The findings reported here are consistent with a potential pathogenic mechanism for FHC whereby acute adrenergic challenge of hearts with chronic low driving force for ATPase reactions leads to cardiac decompensation. If this is the case, the results reported here suggest that lethality associated with FHC need not be due solely to ventricular hypertrophy, but may also result from discrete alterations in the regulation of myofilament activation at the level of the cardiac sarcomere. These changes may well contribute to the broad range of clinical phenotypes that have been observed in patients with cTnT-related FHC.

\section{Acknowledgments}

M.M. Javadpour was supported by NIH grant T32 HL10435, J.C. Tardiff by K08 HL-68619, and J.S. Ingwall by SCOR HL-53230 and R01-HL-63985.

1. Maron, B.J., Bonow, R.O., Cannon, R.O., 3rd, Leon, M.B., and Epstein, S.E. 1987. Hypertrophic cardiomyopathy. Interrelations of clinical manifestations, pathophysiology, and therapy. N. Engl. J. Med. 316:780-789.

2. Towbin, J.A. 2000. Molecular genetics of hypertrophic cardiomyopathy. Curr. Cardiol. Rep. 2:134-140.

3. Tobacman, L.S., et al. 2002. The troponin tail domain promotes a conformational state of the thin filament that suppresses myosin activity. J. Biol. Chem. 277:27636-27642.

4. Maytum, R., Geeves, M.A., and Lehrer, S.S. 2002. A modulatory role for the troponin $\mathrm{T}$ tail domain in thin filament regulation. J. Biol. Chem. 277:29774-29780.

5. Greenfield, N.J., Palm, T., and Hitchcock-DeGregori, S.E. 2002. Structure and interactions of the carboxyl terminus of striated muscle alphatropomyosin: it is important to be flexible. Biophys. J. 83:2754-2766.

6. Palm, T., Graboski, S., Hitchcock-DeGregori, S.E., and Greenfield, N.J. 2001. Disease-causing mutations in cardiac troponin T: identification of a critical tropomyosin-binding region. Biophys. J. 81:2827-2837.

7. Maytum, R., Westerdorf, B., Jaquet, K., and Geeves, M.A. 2003. Differential regulation of the actomyosin interaction by skeletal and cardiac troponin isoforms. J. Biol. Chem. 278:6696-6701.

8. Gomes, A.V., Guzman, G., Zhao, J., and Potter, J.D. 2002. Cardiac troponin $\mathrm{T}$ isoforms affect the $\mathrm{Ca} 2+$ sensitivity and inhibition of force development. Insights into the role of troponin $\mathrm{T}$ isoforms in the heart. J. Biol. Chem. 277:35341-35349.

9. Robinson, P., et al. 2002. Alterations in thin filament regulation induced by a human cardiac troponin $\mathrm{T}$ mutant that causes dilated cardiomyopathy are distinct from those induced by troponin $\mathrm{T}$ mutants that cause hypertrophic cardiomyopathy. J. Biol. Chem. 277:40710-40716.

10. Hinkle, A., and Tobacman, L.S. 2003. Folding and function of the troponin tail domain. Effects of cardiomyopathic troponin T mutations. J. Biol. Chem. 278:506-513.

11. Tian, R., and Ingwall, J.S. 1996. Energetic basis for reduced contractile reserve in isolated rat hearts. Am. J. Physiol. 270:H1207-H1216.

12. Tian, R., et al. 1997. Role of MgADP in the development of diastolic dysfunction in the intact beating rat heart. J. Clin. Invest. 99:745-751.

13. Tian, R., Nascimben, L., Ingwall, J.S., and Lorell, B.H. 1997. Failure to maintain a low ADP concentration impairs diastolic function in hypertrophied rat hearts. Circulation. 96:1313-1319.

14. Ingwall, J., and Neubauer, S. 1999. Heart Failure Reviews: The Energetics of the Failing Heart. 6-2-1999. Kluwer Academic Publishers. Norwell, Massachusetts, USA

15. Jung, W.I., et al. 1998. 31P NMR spectroscopy detects metabolic abnormalities in asymptomatic patients with hypertrophic cardiomyopathy. Circulation. 97:2536-2542.

16. Spindler, M., et al. 1998. Diastolic dysfunction and altered energetics in the $\alpha \mathrm{MHC}^{403 /+}$ mouse model of familial hypertrophic cardiomyopathy. J. Clin. Invest. 101:1775-1783.

17. Tardiff, J.C., et al. 1999. Cardiac troponin T mutations result in allelespecific phenotypes in a mouse model for hypertrophic cardiomyopathy. J. Clin. Invest. 104:469-481.

18. Ingwall, J.S., Saupe, K., and Spindler, M. 2002. Cardiovascular physiology in the genetically engineered mouse. 2 nd edition. B.D. Hoit and R.A. Walsh, editors. Kluwer Academic Publishers. Norwell, Massachusetts, USA. 183-192.

19. Chu, G., et al. 1996. Compensatory mechanisms associated with the hyperdynamic function of phospholamban-deficient mouse hearts. Circ. Res. 79:1064-1076.

20. Saupe, K.W., Spindler, M., Tian, R., and Ingwall, J.S. 1998. Impaired 
cardiac energetics in mice lacking muscle-specific isoenzymes of creatine kinase. Circ. Res. 82:898-907.

21. Ingwall, J.S. 1982. Phosphorus nuclear magnetic resonance spectroscopy of cardiac and skeletal muscles. Am. J. Physiol. 242:H729-H744.

22. Lowry, O.H., Rosebrough, N.J., Farr, A.L., and Randall, R.J. 1951. Protein measurement with the folin phenol reagent. J. Biol. Chem. 193:265-275.

23. Kammermeier, H. 1973. Microassay of free and total creatine from tissue extracts by combination of chromatographic and fluorometric methods. Anal. Biochem. 56:341-345.

24. Rosalki, S.B. 1967. An improved procedure for serum creatine phosphokinase determination. J. Lab. Clin. Med. 69:696-705.

25. Oblinger, M.M., Foe, L.G., Kwiatkowska, D., and Kemp, R.G. 1988. Phosphofructokinase in the rat nervous system: regional differences in activity and characteristics of axonal transport. J. Neurosci. Res. 21:25-34.

26. Bernstein, L.H., and Everse, J. 1975. Determination of the isoenzyme levels of lactate dehydrogenase. Methods Enzymol. 41:47-52.

27. Bergmeyer, H.U., and Gawehn, K. 1974. Methods of enzymatic analysis. Academic Press. New York, New York, USA. 466-467.
28. Polimeni, P.I., and Buraczewski, S.I. 1988. Expansion of extracellular tracer spaces in the isolated heart perfused with crystalloid solutions: expansion of extracellular space, trans-sarcolemmal leakage, or both? J. Mol. Cell. Cardiol. 20:15-22.

29. Lawson, J.W., and Veech, R.L. 1979. Effects of $\mathrm{pH}$ and free $\mathrm{Mg} 2+$ on the Keq of the creatine kinase reaction and other phosphate hydrolyses and phosphate transfer reactions. J. Biol. Chem. 254:6528-6537.

30. Veech, R.L., Lawson, J.W., Cornell, N.W., and Krebs, H.A. 1979. Cytosolic phosphorylation potential. J. Biol. Chem. 254:6538-6547.

31. Ingwall, J.S. 2002. ATP and the heart. Kluwer Academic Publishers. Norwell, Massachusetts, USA. 264 pp.

32. Sweeney, H.L., Feng, H.S., Yang, Z., and Watkins, H. 1998. Functional analyses of troponin $\mathrm{T}$ mutations that cause hypertrophic cardiomyopathy: insights into disease pathogenesis and troponin function. Proc. Natl. Acad. Sci. U. S. A. 95:14406-14410.

33. Knollmann, B.C., et al. 2001. Inotropic stimulation induces cardiac dysfunction in transgenic mice expressing a troponin $\mathrm{T}(\mathrm{I} 79 \mathrm{~N})$ mutation linked to familial hypertrophic cardiomyopathy. J. Biol. Chem. 276:10039-10048. 